\title{
Efficient Video Transfer using LAN Caching assisted by Cloud Computing
}

\author{
Vishwa Kiran S \\ Dept. of Computer Science and Engineering, \\ University Visvesvaraya College of Engineering, \\ Bangalore, India \\ Email: nimmakiran@yahoo.com \\ Thriveni J \\ Dept. of Computer Science and Engineering, \\ University Visvesvaraya College of Engineering, \\ Bangalore, India
}

\author{
Raghuram S \\ Pushkala Technologies Pvt Ltd, \\ Bangalore, India
}

\author{
Venugopal K R \\ Dept. of Computer Science and Engineering, \\ University Visvesvaraya College of Engineering, \\ Bangalore, India
}

\begin{abstract}
There is a good probability of accessing same video content multiple times from a cloud based Video Streaming Server by same peer or different peers of a given LAN, effectively increasing Internet bandwidth or data flow for same content from server to client, thereby over loading routers between server and client and also resulting in higher power consumption at routers. This proposed concept tries to avoid multiple streaming of high volume video files from Server by caching first successful streamed data on to LAN peer which is currently viewing the video data and subsequently the same LAN peer streaming the video to other desiring peers when demanded for. Proposed implementation model retains all other server activities with server except for allowing an available LAN peer copy of video to be streamed to another peer of the same LAN when requested for.
\end{abstract}

Keywords-Cloud Video Server; LAN Caching; Peer to Peer; Router Power Saving; Video Caching; Video Stream Server;

\section{INTRODUCTION}

Multimedia and Internet revolution has brought in many avenues for publishing video and animated content by individuals and business organizations on large scales. Video streaming protocols like RTSP [1] have paved the way for sophisticated video streaming servers like YouTube. Due to ever growing user base and repeated viewing of video contents, streaming servers are getting increasingly overburdened with respect to bandwidth and computing capabilities. Commercial streaming servers can very well sustain the effect, but social enterprise and academic servers like Coursera [2], MIT Open Course [3] and National Programme on Technology Enhanced Learning (NPTEL) [4] could feel the pinch.

Collection of demographic information, view analysis, collecting user feedback, streaming overlapping or intermittent advertisements and such related activities are holding back the options of download and share possibilities being provided by Video Streaming Severs (VSS).
This work is intended to provide an optimized solution to cache video content on LAN peers for repeated usage, still allowing VSS to collect demographic information, process and analyze feedbacks and stream live advertisements.

\section{A. Motivation:}

NPTEL [4] is a project propounded and funded by Ministry of Human Resource and Development, Government of India. NPTEL is aiming at promoting and imparting quality science and technology content to interested students globally. Seven IITs and IISc are participating members of NPTEL and have contributed majority of teaching contents as video lectures. These video lectures are viewed globally and majority of the viewers are from India. Fig. 1, Fig. 2 and Fig. 3 [4] depicts the viewing statistics at the time of compilation of this paper. These statistics are directly derived from NPTEL website.

It's learnt from NPTEL that, more than 18200 video lectures are made available through NPTEL's YouTube channel and more than 395,000 viewers have subscribed to the channel. To this date viewings has surpassed 120 million. This kind of data gathering or analysis is possible due to cloud infrastructure and video streaming servers.

This technology scheme demands for heavy Internet bandwidth. Given an academic institute environment bandwidth demands are even more due to the fact that, hundreds of PCs, laptops are hooked up to a single Internet channel or route. This limitation of heavy Internet bandwidth requirement and possibility of repeated viewing of same videos has motivated us to explore and exploit the possibility of caching videos on LAN peers and reuse when necessary.

\section{B. Contribution}

This work aims to reduce Internet bandwidth by caching frequently accessed Cloud Server streamed video content with in LAN peer/s, and use it for subsequent viewing by itself or other peers in same LAN. 


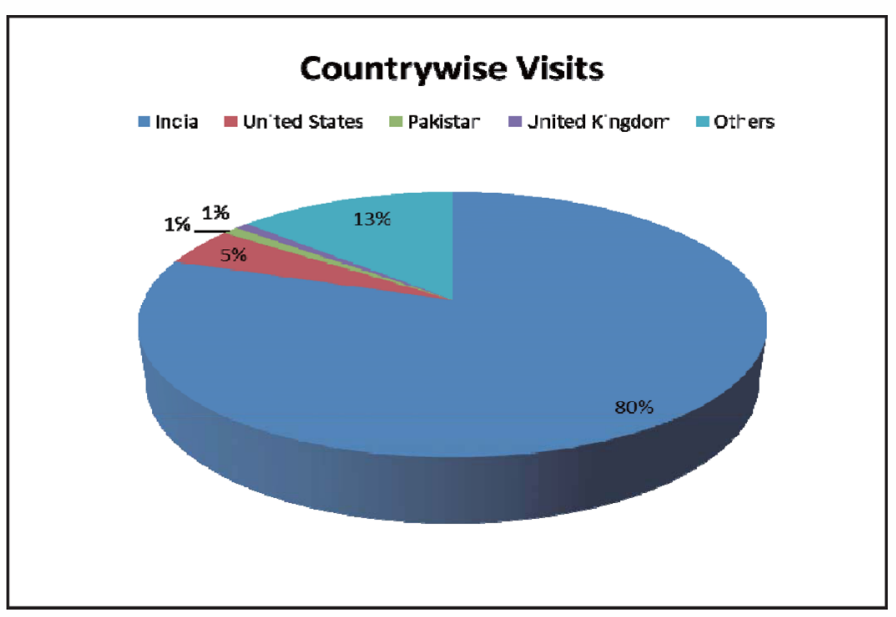

Fig. 1. Video lectures watched through NPTEL video streaming server

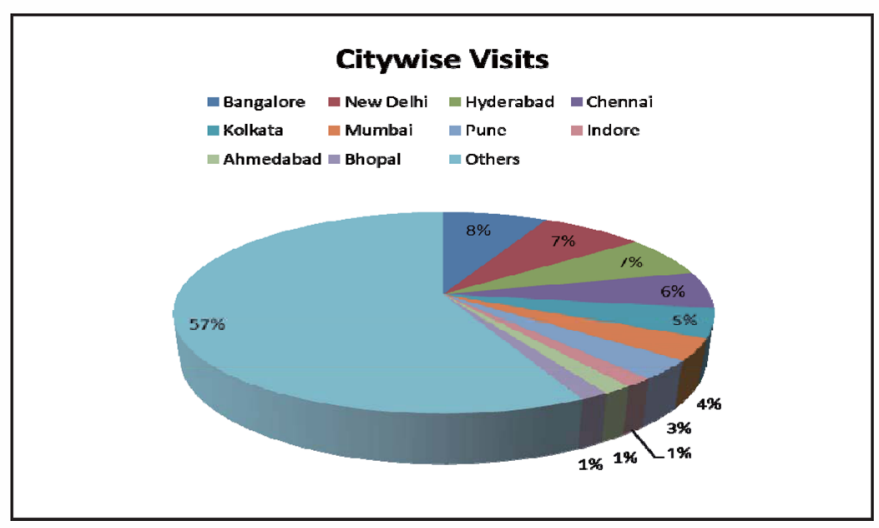

Fig. 2. Indian cities breakup of video lectures watched through NPTEL video streaming server.

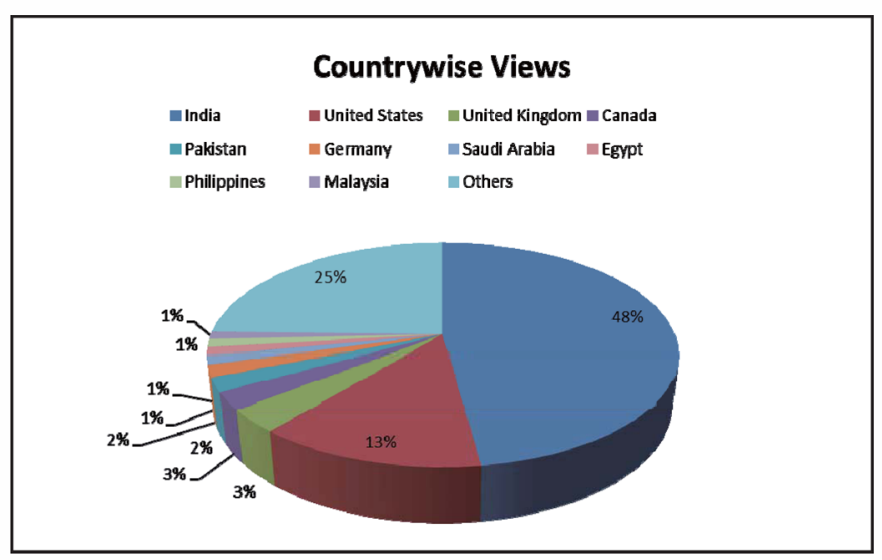

Fig. 3. Video lectures watched through YouTube.

\section{Organization}

Section II discusses about Literature Survey, proposed architecture of VSS and LAN Peer video streaming is explained in Section III, results are presented in Section IV and conclusions are drawn in Section V.

\section{LITERATURE SURVEY}

In the past there has been similar work [4] to measure viewing pattern of YouTube and provide local and peer caching facilities. A measurement study [5] and simulation result of related work has encouraged us to explore possibilities of developing LAN peer cached solutions to augment VSS services. Software utilities like FFMPEG Server and others [6] help making solution easier. There has been a previous effort [7] in evaluating efficiency of peer-to-peer video streaming, and if clients are mobile in nature, many adaptive and predictive models has also been suggested and evaluated [8]-[12].

There could be scenarios of clogged WLANs that may hinder good quality streaming video service through peers. These situations are also been addressed by researchers and newer efficient models [13] have been proposed in the recent past.

In large scale LAN environments like college or tutorial classes or community centers there could be interference to WLAN infrastructure by radio units operating in the same frequency band. Example being a WiFi router interfered by a Bluetooth PAN. Author Antonios Argyriou in his work [14] suggests switching from unicast to multicast mode of video streaming to improve efficiency and reliability of video streaming in such an interfering environment. Hence, if there are requests for same video file from multiple peers, a single peer could efficiently multicast (if network setting permits) to all desiring peers. This approach is better suited than scaling of peer streaming servers [15] and further clogging the network.

From power saving perspective there has been many simulation and actual measurement works carried out by various teams. Joule per Bit [16] is the important unit of measurement considered in quantifying power consumption by Routers and Switches in computer network domain. Reducing Joule per Bit [17] \& [18] is the most researched topic in networking domain as energy prices are inflating every day. It is also to be noted that just by reducing network traffic load on the Router does not save power and improve efficiency [19] of the same. There has to be optimal utilization of Routers capacity to improve efficiency and save power eventually.

\section{ARCHITECTURE}

More often a single IP address is shared among all LAN members and needless to say Internet bandwidth is shared among all members of LAN. In scenarios where multiple members try to access media rich content from web bandwidth gets choked and a few times results in compromising streaming quality of media. To circumvent this problem, we intend to propose a solution wherein first time accessed media content from Internet through VSS would be cached on to that particular device of LAN and streamed from it in case of any other local device request for same content, not compromising the advertisement and demographic mechanism of VSS. To test the proposed solution, a VSS and 
LAN environment has been envisaged and its architecture is as shown in the Fig. 4.

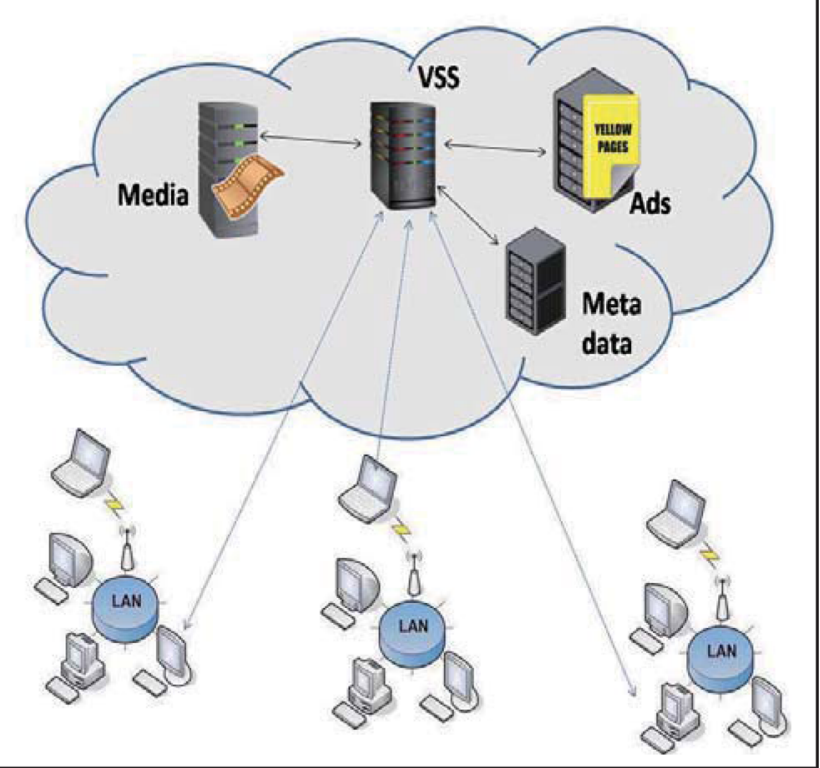

Fig. 4. Architecture of Internet Video Stream Server

LAN clients or peers are termed as consumers and its data exchange mechanism is presented in a flow chart as shown in Fig. 5. Following is brief description of events or data exchanged between consumer and VSS. Any consumer desiring to view a video content available on a specific VSS makes a request through normal Internet browser activity. VSS responds with video links as search result based on the keyword searched by the consumer. Then consumer selects a video link interested to view from the list of videos displayed by the server.

Apart from VSS receiving or registering the selection, same video link or a key representing that link is broadcasted to the LAN. If the same video represented by key is found in the LAN, then stream request is made to LAN peer and the request is made to VSS to send metadata, control information and sync data only. If requested video not to found among LAN peers, then the video content is streamed from VSS. Once the video is successfully streamed by VSS, the consumer will check if the streamed video could be cached, if so, then the content is cached in the consumer machine. In both scenarios, any meta-data or overlapping content like advertisement is always streamed from VSS and it is synchronized with actual video content and rendered on browser.

Flow chart for Video Streaming Server activities is as shown in the Fig. 6. VSS will be waiting for the client's request for streaming a video. Once search request is received it generates video link content for viewing and waits for video link selection. Two types of link selection request could be received by VSS, as mentioned in consumer flow. One with video data and all meta-information, and the other only for meta-information where, video is steamed from one of LAN peer. Based on type of request VSS takes appropriate decision to stream only meta-data or bundled with video.

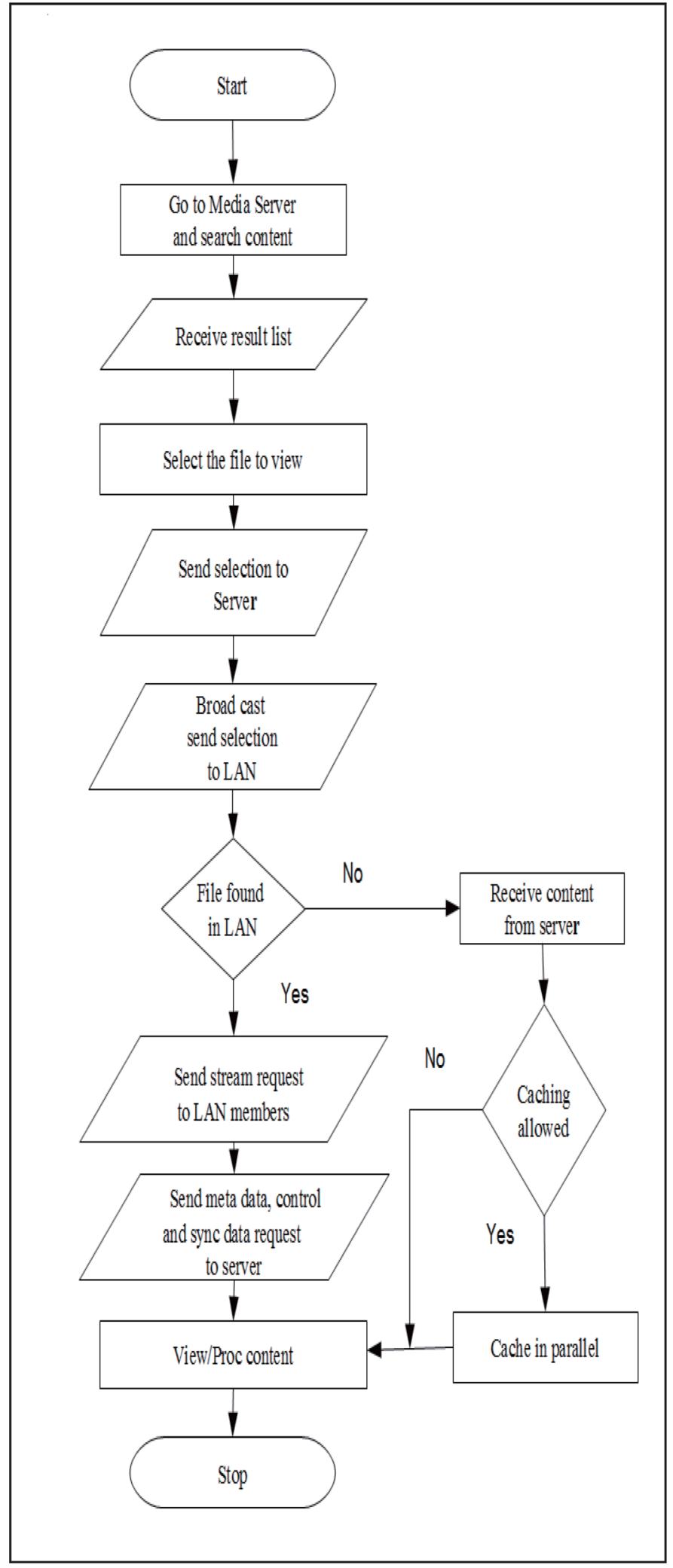

Fig. 5. Flow Chart for Consumer

As proposed VSS activities are not readily available in referred [2] and [3] Video Streaming Servers, Both Internet or Cloud based VSS and consumer end local video streaming 
entity using FFMPEG server and client modules are proposed in this work. NPTEL contents are legally available to be reused in academic institutions hence our initial trails are focused towards caching NPTEL contents only

No
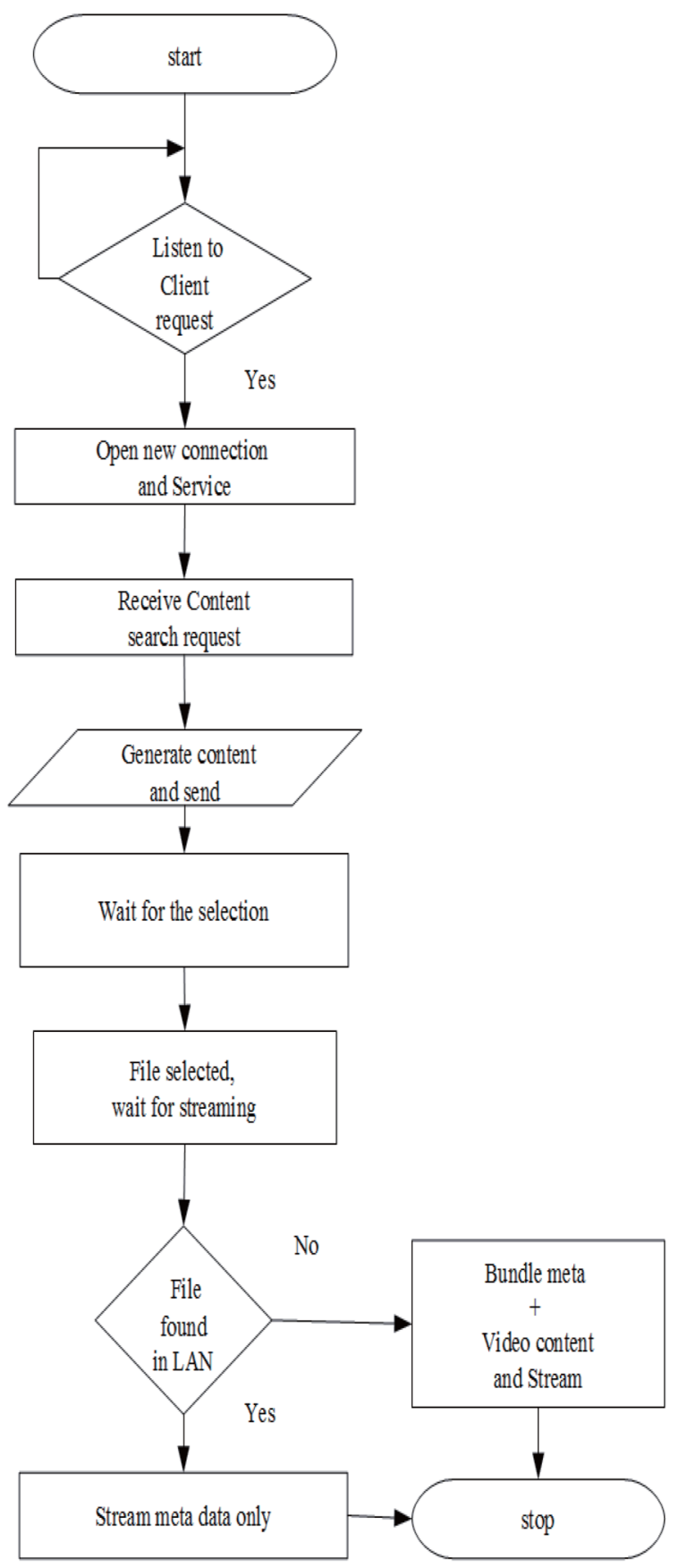

Fig. 6. Flow Chart for Server

\section{RESULTS}

Experiments are conducted to prove or hypothesis that there is a good possibility of repeated viewing of same video material by different viewers in a LAN environment. Following are the results.

\begin{tabular}{|l|l|}
\hline Total number of browser considered & 46 \\
\hline Total number of history links sampled links & 77352 \\
\hline Total number of YouTube video watch links & 3626 \\
\hline $\begin{array}{l}\text { Total number of YouTube links which are viewed } \\
\text { more than once }\end{array}$ & 129 \\
\hline Highest view count of a YouTube link & 6 \\
\hline Total number of NPTEL video watch links & 68 \\
\hline $\begin{array}{l}\text { Total number of NPTEL links which are viewed } \\
\text { more than once }\end{array}$ & 5 \\
\hline Highest view count of a NPTEL link & 4 \\
\hline
\end{tabular}

History links samples are more than 18 months old, and dating up to the time of compilation of this paper. Python script was developed to parse the history links and yield the results. NPTEL related data obtained is too small to process and hence we intend to focus more on YouTube data. Once again YouTube links are not filtered to bear only NPTEL related links but includes all video links viewable through YouTube.

Fig. 7, depicts out of 3626 YouTube video watched links, 129 links have been viewed at least twice. Maximum views range up to 6 , and views evidently average to 2.33 views per link. As seen from the graph, viewing peeks take a grouped structure and calls for further investigation.

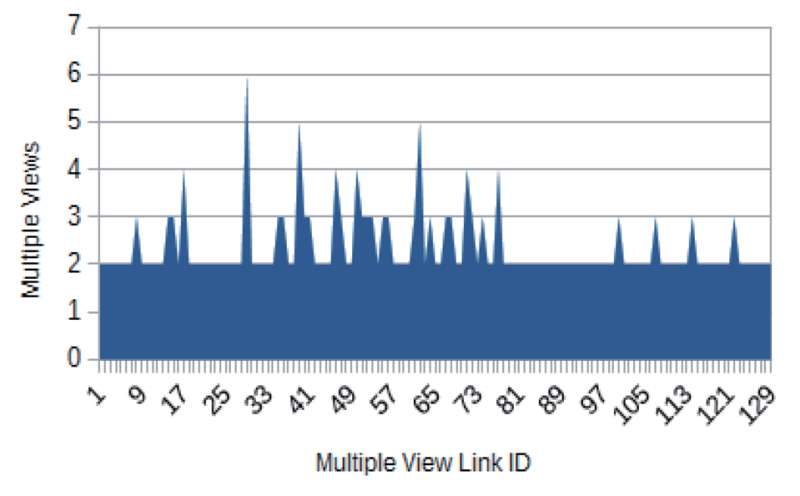

Fig. 7. Multiple view statistics of YouTube videos.

Fig. 8 depicts multiple view sample distribution over available 3626 samples. Graph's non linearity(x axis represents link one of 129 and y axis represents links one of 3626 links) clearly indicates that multiple viewing pattern concentrates during some period of samples and is not same over the complete 18 months of our sample duration. This could be correlated to academic calendar viewership that could easily decline during tests or exams and increase during normal academic activity days.

These initial promising results are demanding for further investigation of the same from various and more statistical 
analytical methods. Hence, we indent to continue work with growing samples and by applying more formal methods.

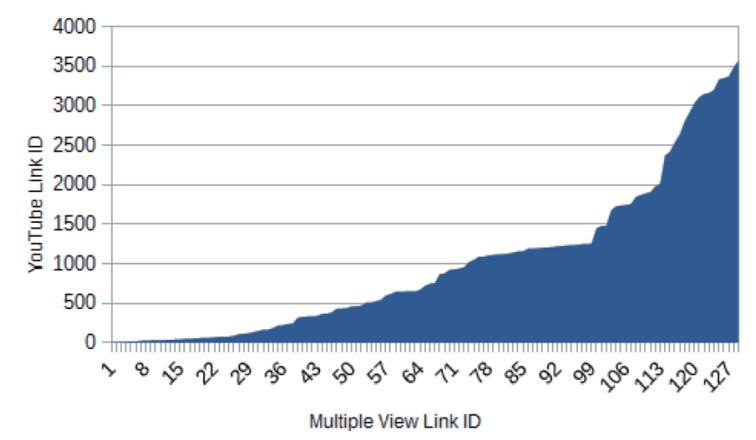

Fig. 8. Multiple view link sample distribution.

It is emphasized that through successful implementation of proposed work saves Internet bandwidth by caching video content. Routers efficiency will not be compromised due to the fact that routers are already overloaded and their capacity are scaled through cloud computing algorithms.

Consider an example from our results, multiple viewed YouTube Video link sample number 29 has been viewed totally 6 times, its duration is 2 minutes and 34 seconds, streaming size when viewed with mp4 HD quality is $26,432,134$ bytes. Since first view is always from VSS, hence Internet bandwidth saved is $\mathrm{N}-1$, Where $\mathrm{N}$ is total multiple view count. In this particular case, bandwidth saved is $26,432,134 \times 5=132,160,670$ bytes. This could be approximated to $132 \mathrm{M}$ bytes of saving.

From power saving perspective if the same 132M bytes of data is streamed through a single router based LAN, it would be saving at least 7 times the power when compared to an 8 router hop based video streamed through a cloud server. Here we have considered 8 hops as average number of hops between client and server.

\section{CONCLUSION}

Our hypothesis of multiple viewing of same video material by different LAN peers or browsers in a LAN environment has been proved correct by our initial experimental data presented. Apart from obvious saving of Internet bandwidth and congestion avoidance, the proposed solution improves the quality of media streaming by multiple folds. Compared to an average $2 \mathrm{Mbps}$ Internet bandwidth, WIFI LAN based 50 Mbps bandwidth connectivity provides jitter free streaming of full HD quality video content without manual buffering. The proposed work is put to further analysis and development, so as to get substantial evidence that the hypothesis is proved correct.

\section{REFERENCES}

[1] "RFC - Real Time Streaming Protocol", http:// www.ietf.org/rfc/rfc2326.txt

[2] "Online Free Courses ", http://www.coursera.org

[3] "Open Course Ware - Massachusetts Institute of Technology ", http://ocw.mit.edu

[4] "National Programme on Technology Enhanced Learning", http://nptel.iitm.ac.in

[5] Michael Zink, Kyoungwon Suh, Yu Gu and Jim Kurose, "Watch Global Cache Local: YouTube Network Traffic at a Campus Network Measurements and Implications", Proc. SPIE6818, Multimedia Computing and Networking, 2008.

[6] Kemal Deniz Teket, Muge Sayit and Geylani Kardas, "Software agents for peer-to-peer video streaming", IET Software., Vol. 8 Iss. 4, pp. 184192, 2014.

[7] Shafquat Ali Memon, Syed Raheel Hassan and Nisar Ahmed Memon, "Evaluation of Video Streaming Performance over Peer-to-Peer Network", International Conference on Collaboration Techonologies and Systems(CTS), 2014.

[8] Bin Pan, Xiaofei Wang, Chung-Pyo Hong and Shin-Dug Kim, "AMVPCloud: A Framework of Adaptive Mobile Video Streaming and User Behaviour Oriented Video Pre-fetching in the Clouds", IEEE $12^{\text {th }}$ International Conference on Computer and Information Technology, 2012.

[9] Yan Liu and Jack Y. B. Lee, "On Adaptive Video Streaming with Predictable Streaming Performance", IEEE Global Communication Conference, 2014.

[10] Xiaofei Wang, Min Chen, Kwon T T, Yang L T and Leung V C M, "AMES-Cloud Mobile Video Streaming and Efficient Social Video Sharing in the Clouds, IEEE Transactions on Multimedia, Vol. 15. No. 4, 2013.

[11] Jaehyun Hwang, Junghwan Lee, Nakjung Choi and Yoo C, "HAVS: Hybrid Adaptive Video Streaming for Mobile Devices", IEEE Transactions on Consumer Electronics, Vol. 60, No. 2, 2014.

[12] Yan Liu and Lee J Y B, "Providing Predictable Streaming Performance in Mobile Video Streaming", IEEE International Conference on Communicatins(ICC), 2014.

[13] Yao X W, Wang W L and Yang S H, "Video Streaming Transmission: Performance Modelling over Wireless Local Area Networks under Saturation Condition", IET Communications, Vol. 6, No. 1, 2012.

[14] Antonios Argyriou, "Compressed Video Streaming in Cooperative Wireless Networks with Interfacing Transmissions", IEEE Transactions on Communications, Vol. 60. No.11, 2012.

[15] Kianoosh Mokhtarian and Mohamed Hefeeda, "Capacity Management of Seed Servers in Peer-to-Peer Streaming Systems With Scalable Video Streams", IEEE Transactions on Multimedia, Vol. 15. No. 1, 2013.

[16] Jon Dugan, "Network Power Measurement at ESnet", US Department of Engergy, 2013

[17] Kerry Hinton, Jayant Baliga, Michael Feng, Robert Ayre and Rodney S Tucker, "Power Consumption and Energy Efficiency in the Internet", IEEE Network, 2011

[18] Joseph Chabarek, Joel Sommers, Paul Barford, Cristian Estan, David Tsiang and Steve Wright, "Power Awareness in Network Design and Routing", In Procedings of IEEE INFCOM, 2008.

[19] "Measurement based network element power modeling", http://gt-gointworkshop.fp7-trend.eu 